\title{
Placas alinhadoras uma opção estética no tratamento ortodôntico: revisão de
}

\section{literatura}

\author{
Alignment plates an aesthetic option in orthodontic treatment: literature review \\ Las placas de alineación una opción estética en el tratamiento de ortodoncia: revisión de la
}

literatura

Recebido: 21/07/2021 | Revisado: 29/07/2021 | Aceito: 05/08/2021 | Publicado: 09/08/2021

Danival Roberto Medeiros Júnior

ORCID: https://orcid.org/0000-0002-8774-6256

Faculdade Patos de Minas, Brasil

E-mail: juniordanival@gmail.com

Amanda Patrícia Silva

ORCID: https://orcid.org/0000-0003-3709-7988

Faculdade Patos de Minas, Brasil

E-mail: amandatiros81@gmail.com

Cláudia Maria de Oliveira Andrade

ORCID: https://orcid.org/0000-0003-4529-8106 Faculdade Patos de Minas, Brasil

E-mail: claudiamoacd@yahoo.com.br

Victor da Mota Martins

ORCID: https://orcid.org/0000-0001-6631-6161

Centro Universitário de Patos de Minas, Brasil E-mail: victortag@hotmail.com

Lia Dietrich

ORCID: https://orcid.org/0000-0001-7887-8591

Universidade Federal do Vale do Jequitinhonha e Mucuri, Brasil

E-mail: lia_dietrich@yahoo.com.br

\begin{abstract}
Resumo
Atualmente os padrões estéticos vem aumento a cada dia, a sociedade criou um padrão de beleza ideal no qual, dentes brancos, alinhados, nivelados e com o formato que julgam belo é indicativo de saúde bucal. Tal fato faz com que a procura por profissionais da Odontologia estética acabe aumentando, em especial a busca pelos ortodontistas. Entretanto os aparelhos ortodônticos fixos convencionais não são considerados estéticos, o que acabou forçando a evolução desse aspecto dentro da ortodontia.Após muitos estudos desenvolveu-se e aprimorou-se os alinhadores invisíveis, considerados padrão ouro no quesito estética, estes se mostram eficientes e apresentam uma boa previsibilidade quando empregados dentro de suas indicações. Este trabalho objetiva evidenciar os alinhadores invisíveis como mais uma opção dentro de seu campo de trabalho. Foi realizada uma revisão de literatura narrativa qualitativa, utilizando-se como base bancos de dados PubMED, Lillacs, BVSsalud e Scielo, nas línguas portuguesa e inglesa, sem restrição de ano. Foi observado que as indicações para esses alinhadores invisiveis ainda são extremamente restritas, em muitos casos os alinhadores não conseguem resultados tão satisfatórios quanto os aparelhos fixos convencionais e quando conseguem necessitam de um maior tempo de tratamento, fato este que acarreta em maiores custos.
\end{abstract}

Palavras-chave: Odontologia; Estética; Estética dentária; Ortodontia; Aparelhos ortodônticos removíveis.

\begin{abstract}
Currently, aesthetic standards are increasing every day, society has created an ideal beauty standard in which white teeth, aligned, leveled and with the shape they deem beautiful is indicative of oral health. This fact means that the search for professionals in cosmetic dentistry ends up increasing, especially the search for orthodontists. However, conventional fixed orthodontic appliances are not considered aesthetic, which forced the evolution of this aspect within orthodontics. After many studies, invisible aligners were developed and improved, considered the gold standard in terms of aesthetics, they are efficient and have good predictability when used within their indications. This work aims to highlight the invisible aligners as another option within its field of work. A qualitative narrative literature review was carried out, using PubMED, Lillacs, BVSsalud and Scielo databases, in Portuguese and English, with no year restrictions. It was observed that the indications for these invisible aligners are still extremely restricted, in many cases the aligners do not get results as satisfactory as conventional fixed appliances and when they do, they need a longer treatment time, which results in higher costs.
\end{abstract}

Keywords: Dentistry; Esthetics; Esthetics dental; Orthodontics; Orthodontic appliances removable. 


\begin{abstract}
Resumen
Actualmente, los estándares estéticos están aumentando día a día, la sociedad ha creado un estándar de belleza ideal en el que los dientes blancos, alineados, nivelados y con la forma que consideran hermosa es indicativo de salud bucal. Este hecho hace que la búsqueda de profesionales en odontología cosmética acabe aumentando, especialmente la búsqueda de ortodoncistas. Sin embargo, los aparatos de ortodoncia fijos convencionales no se consideran estéticos, lo que obligó a la evolución de este aspecto dentro de la ortodoncia. Después de muchos estudios, se desarrollaron y mejoraron los alineadores invisibles, considerados el estándar de oro en términos de estética, son eficientes y tienen una buena predictibilidad cuando se utilizan dentro de sus indicaciones. Este trabajo tiene como objetivo destacar los alineadores invisibles como una opción más dentro de su campo de trabajo. Se realizó una revisión de literatura narrativa cualitativa, utilizando las bases de datos PubMED, Lillacs, BVSsalud y Scielo, en portugués e inglés, sin restricciones de año. Se observó que las indicaciones de estos alineadores invisibles aún son sumamente restringidas, en muchos casos los alineadores no obtienen resultados tan satisfactorios como los aparatos fijos convencionales y cuando lo hacen, necesitan un tiempo de tratamiento mayor, lo que se traduce en mayores costos.
\end{abstract}

Palabras clave: Odontología; Estética; Estética dental; Ortodoncia; Aparatos ortodônticos removibles.

\title{
1. Introdução
}

A importância da estética exigida pelas pessoas na sociedade moderna acabou criando um padrão de beleza, no qual é considerado um indicador de saúde bucal a posse de dentes brancos, bem alinhados, nivelados com um formato ideal. Tal fato acabou gerando uma procura por ortodontistas, com o objetivo de corrigir discrepâncias dentarias e esqueléticas (Couto \& Abreu, 2020). A aparência dos dentes influencia diretamente na saúde mental e comportamento do indivíduo, apresentando potencial para causar diversas restrições sociais, profissionais e afetivas (Couto \& Abreu, 2020; Phan \&Ling, 2007).

O uso de aparelhos ortodônticos fixos convencionais, acaba gerando um desconforto no paciente durante o período de tratamento, uma vez que, estes são compostos por bandas, bráquetes, fios e ligaduras. Estas características dos materiais acabaram obrigando a ortodontia a evoluir, procurando alcançar a tão desejada estética (Couto \& Abreu, 2020; Phan \&Ling, 2007).

A princípio, a solução para melhorar a estética, foi o desenvolvimento de aparelhos fixos com bráquetes de porcelana policristalina ou monocristalina, entretanto, não foi uma solução definitiva, uma vez que, os pacientes desejavam aparelhos invisíveis, os quais passassem despercebidos por terceiros (Couto \& Abreu, 2020; Vieira, Franco, \& Guimarães Junior, 2013). Com isso, os alinhadores invisíveis acabaram se tornando um grande aliado (Couto \& Abreu, 2020).

Os indivíduos que procuram por tratamentos ortodônticos estéticos, são adultos motivados e preocupados com sua aparência, a qual foi gerada pela pressão da sociedade e seu padrão de beleza ideal (Neves, Coutinho, Ferreira, Coutinho, \& Miranda, 2012; Phan \& Ling, 2007).

O primeiro relato de posicionador dental removível foi descrito por Kesling em 1945, feito com base em setup individualizado de modelos de gesso, e desde então, outros dispositivos foram desenvolvidos até chegar aos modelos atuais (Jóias, Sanders, Cepera, Paranhos, \& Torres, 2011).

Atualmente os alinhadores são criados por um software de computador, necessitam de apenas uma moldagem, requerem poucos exames complementares e possibilitam uma excelente previsibilidade do tratamento, quando utilizados dentro de suas indicações (Couto \& Abreu, 2020; Phan \& Ling, 2007). Possuem um nível estético satisfatório, se mostram confortáveis, de fácil higienização, provocam menos traumas quando comparados aos aparelhos fixos convencionais, são menos incômodos para os pacientes uma vez que podem ser removidos e colocados de volta sem dificuldade pelo próprio paciente, e demonstram resultados excelentes em apinhamentos leves e moderados (Couto \& Abreu, 2020; Phan \& Ling, 2007; Neves et al, 2012). Em casos mais complexos eles permitem a implementação de acessórios que aumentam a sua efetividade, no entanto, não são tão indicados, pois não atingem resultados efetivos, fazendo com que os aparelhos fixos convencionais continuem sendo os mais indicados para a realização destes tratamentos (Couto \& Abreu, 2020; Phan \& Ling, 2007). Seu uso também é limitado no tempo de tratamento, visto que apresenta como efeito colateral a mordida aberta dos dentes posteriores (Phan \& Ling, 2007; Neves et al, 
2012). Além disso, podem ter seu efeito prejudicado pelo fato de negligência por parte dos pacientes por não utilizar pelo tempo ideal de 22 horas por dia. Podem ser danificados, perfurados ou quebrados, em especial pelos pacientes que apresentam parafunções (Phan \& Ling, 2007).

Estes alinhadores fazem uso de alta tecnologia, através de um "projeto assistido por computador" (CAD, em inglês, computer-aided design) e "fabricação assistida por computador", (CAM, em inglês, computer-aided manufacturing) para realizar a previsão dos resultados do tratamento ortodôntico e então produzir diversos alinhadores personalizados, com uma sequência para realizar seu uso, estes são produzidos com um material plástico transparente, possuindo uma espessura de aproximadamente 1 milímetro que recobre toda a coroa dentária e a gengiva marginal (Couto \& Abreu, 2020; Phan \& Ling, 2007).

Cada alinhador possui a capacidade de realizar uma movimentação dentaria de 0,25 a 0,30 milímetros dentro de um período de aproximadamente duas semanas (Couto \& Abreu, 2020). Os tratamentos ortodônticos com alinhadores invisíveis, necessitam da realização sucessiva de trocas, seguindo uma ordem especifica, a qual depende de cada caso e da colaboração de cada paciente. O paciente é um dos principais responsáveis pelo tratamento e pode influenciar diretamente na eficácia do mesmo, pois é necessário realizar o uso dos alinhadores por 22 horas diárias para que ao final de 2 semanas se totalizem 400 horas de uso antes de passar para o próximo alinhador (Jóias et al, 2011; McNamara, Kramer, \&Juenker, 1985; Kesling, 1945; Ponitz, 1971). Quando comparados com os aparelhos ortodônticos fixos convencionais os alinhadores invisíveis acabam favorecendo uma melhor saúde bucal devido a possibilidade de removê-los sempre que for realizar a higienização oral (Couto \& Abreu, 2020; Neves et al, 2012).

Este trabalho objetiva a evidenciação dos alinhadores invisíveis como mais uma opção dentro do campo de trabalho do ortodontista.

\section{Metodologia}

O presente estudo apresenta-se na forma de uma revisão narrativa da literatura, com caráter qualitativo (Estrela, 2018). A pesquisa se deu através da busca nas bases de dados PubMED, Lillacs, BVSsalud e Scielo, contendo palavras chaves como: Odontologia; Estética; Estética dentária; Ortodontia; Aparelhos ortodônticos removíveis, nos idiomas português e inglês, sem restrição de ano. Após essa busca foram selecionados artigos, que abrangiam no tema proposto.

\section{Revisão da Literatura}

\subsection{Placas alinhadoras $x$ ortodontia convencional}

Há muitos anos a eficiência dos tratamentos ortodônticos convencionais foi comprovada (Cardoso, Espinosa, Mecenas, Flores-Mir, \& Normando, 2020; Jóias et al, 2011). Porém, houve um aumento da demanda pela estética, no qual, as pessoas tem buscado cada vez mais tratamentos "invisíveis" e mesmo aqueles aparelhos com bráquetes de porcelana policristalina ou monocristalina acabam não satisfazendo plenamente o quesito "invisibilidade" (Jóias et al, 2011; Vieira et al, 2013). E foi nesse contexto, que os alinhadores ganharam força e se tornaram um tratamento tão procurado (Noble, Hechter, Karaiskos, Lekic, \& Wiltshire, 2009; Vieira et al, 2013). Vale ressaltar que os ortodontistas apresentam boa aceitação, tanto pelos aparelhos convencionais com bráquetes e fios, quanto pelos aparelhos do sistema Invisalign® (Noble et al, 2009).

Quando os alinhadores estéticos surgiram no mercado, acreditava-se que eles seriam capazes de resolver outros problemas do aparelho ortodôntico convencional, tais como desconfortos e lesões provocados por acessórios metálicos, dificuldade de higienização e a dificuldade de mastigar no início do tratamento (Couto \& Abreu, 2020; Cardoso et al, 2020). No entanto, esse tratamento envolve trocas sucessivas de aparelhos a cada 15 dias e necessita da colaboração do paciente, pois é preciso que o ele o utilize por 20 a 22 horas por dia, somando um total de 400 horas por placa (Phan \&Ling, 2007).

No tratamento com Invisalign ${ }^{\circledR}$ é possível que se tenha um maior controle e uma maior previsibilidade no tratamento 
através do sistema CAD/CAM, pelo qual antes mesmo de se começar o tratamento já se sabe qual será o resultado alcançado e o tempo de tratamento. Nos aparelhos fixos, o planejamento é realizado pelo ortodontista e não se tem uma previsibilidade tão boa, tornando o ortodontista, responsável por conduzir o tratamento e controlar todas as movimentações, fazendo ativações a cada mês (Thiesen, 2021).

Boyod, relatou dois casos clínicos no qual ele fez o uso dos dois aparelhos e conseguiu demonstrar que o sistema Invisalign ${ }^{\circledR}$ conseguiu ser eficaz em aproximadamente $70 \%$ do tratamento necessitando do uso dos aparelhos ortodônticos fixos em apenas 30\% do tratamento (Boyod, 2005). Kravitz et al, afirmaram que a Align Technology admite que 20 a 30\% dos seus tratamentos requerem correção de curso ou impressões de refinamento para ajudar a atingir os objetivos de pré-tratamento, mas quando perguntado à ortodontistas eles afirmam que cerca de 70 a $80 \%$ dos tratamentos precisam desses ajustes durante o tratamento (Kravitz et al, 2009).

Em uma revisão sistemática e meta-analise, conseguiu afirmar que em pacientes Classe I de Angle, com apinhamentos menores que $4 \mathrm{~mm}$ e sem extrações, os aparelhos fixos se mostraram mais eficazes na melhora das variáveis que quantificam o resultado pós-tratamento, superando o Invisalign® nos quesitos corredor bucal, linha média, exibição gengival, inclinação do sorriso, linha média maxilar e alto índice do sorriso (o Invisalign ${ }^{\circledR}$ apresentou um baixo índice do sorriso e esse fato pode levar a um fenômeno denominado "envelhecimento do sorriso"), enquanto, o Invisalign® foi capaz de superar os aparelhos fixos apenas na posição e inclinação dos incisivos superiores. Ambos os tratamentos apresentaram bons resultados na largura do sorriso e não apresentaram alterações na simetria e espessura labial (Christou, Abarca, Christou \&Krau, 2020).

Um fator importante que precisamos levar em consideração é a dor dos pacientes durante o tratamento ortodôntico, visto que isso afeta diretamente a qualidade de vida e o medo da dor é um fator que leva algumas pessoas a desistirem do tratamento (Cardoso et al, 2020; Cordeiro, 2020). Nos aparelhos fixos, a dor é mais intensa nas primeiras 24 horas e pode se manter intensa nos 3 primeiros dias, no entanto a dor é minimizada ou desaparece por volta do 7 dia (Cardoso et al, 2020; Cordeiro, 2020; White, Julien, Jacob, Campbell \& Buschang, 2017). Os pacientes tratados com aparelhos fixos relatam sentir mais dificuldade para comer (o que pode ser explicado pelo fato de os pacientes tratados com Invisalign® retirarem o aparelho para se alimentar) e relataram também maior dor nos dentes da frente do que nos dentes de trás (Couto \& Abreu, 2020; White et al, 2017). Nos pacientes tratados com Invisalign (® também temos picos de dores mais altos durante a primeira semana, no entanto esses níveis são menores quando comparados aos aparelhos fixos (Cardoso et al, 2020; White et al, 2017). Em ambos os casos, os níveis de desconforto relatados nos ajustes mensais atingiram picos mais baixos de dor do que após a ligação inicial (White et al, 2017).

Em um estudo realizado por White et al, 2017, cerca de 45\% dos pacientes tratados com aparelhos fixos, utilizaram algum tipo de analgésico nos 2 primeiros dias, enquanto, nos pacientes tratados com o sistema Invisalign ${ }^{\circledR}$ esse número subiu para 56\% nos 2 primeiros dias e depois diminuiu mais rapidamente nos dias seguintes (White et al, 2017).

Pacientes tratados com alinhadores ortodônticos, apresentam uma melhor saúde gengival com diferença significativa nos índices placa e profundidade de sondagem, quando comparados aos pacientes que foram tratados com aparelhos convencionais (Lu, Tang, Zhou, \& Kang, 2018). Esses pacientes também apresentam um número menor de cáries e lesões de manchas brancas (Couto \& Abreu, 2020). O que pode ser explicado pelo fato de os alinhadores serem removidos durante a alimentação e durante processo de higienização, consequentemente causando uma retenção muito menor de alimentos e possibilitando uma melhor higienização (Couto \& Abreu, 2020; Lu et al, 2018). No entanto a longo prazo, não se observa diferenças significativas, pois acredita-se que os pacientes melhoram suas habilidades de higienização com o aparelho fixo. (Chhibber, Agarwal, Yadavm, Kuo, \& Upadhyay, 2018).

Quando se trata do tempo de tratamento, temos diversos estudos envolvendo o tratamento com Invisalign® e o tratamento com aparelhos ortodônticos fixos, e o que se pode observar é que os pacientes tratados com Invisalign® apresentam 
um tempo de tratamento menor (Christou et al, 2020; Cordeiro, 2020). No entanto, vale ressaltar que, estes estudos foram realizados com casos simples, dentro das indicações do Invisalign® ${ }^{\circledR}$, em casos mais complexos o tempo de tratamento com os aparelhos ortodônticos fixos pode ser menor (Cordeiro, 2020).

No que se diz respeito à recidiva ortodôntica, um trabalho avalia o tratamento após o uso da contenção e pode concluir que os casos tratados com Invisalign ${ }^{\circledR}$ apresentaram um maior grau de recidiva, enquanto o outro avaliou as recidivas durante o uso da contenção e não pôde encontrar nenhuma diferença significativa nos dois aparelhos (Santos, 2020).

\subsection{Histórico das placas alinhadoras}

Os primeiros alinhadores ortodônticos foram produzidos por Kesling em 1945, através de um setup individualizado, que era confeccionado a partir de modelos de gesso (Couto \& Abreu, 2020). Nessa época, eles tinham como principal finalidade serem utilizados para o posicionamento artístico final e retenção dos dentes após a realização do tratamento ortodôntico convencional (Kesling, 1945).

Posteriormente as ideias desse autor, foram aperfeiçoadas por Ponitiz em 1971, que introduziu um aparelho chamado "retentor invisível", feito em um modelo mestre que posicionava previamente os dentes numa chapa base de cera (Ponitz, 1971).

McNamara et al, 1985, fizeram algumas melhorias no modelo de Ponitz, 1971, mas não conseguiu apresentar resultados satisfatórios em grandes movimentações. No entanto, ele afirmou que esse aparelho poderia ser usado efetivamente para retenções, permitindo alguns ajustes oclusais de acabamento ou como retentores de transição para manter os dentes em posição até que os aparelhos funcionais apropriados estivessem disponíveis (McNamara, 1985).

Em 1994, foi criado uma técnica que realizava desgastes interproximais e um progressivo alinhamento dentário, porém utilizava o aparelho Essix e ainda eram necessárias novas moldagens e novos setups a cada visita clínica, em um processo trabalhoso e que consumia muito tempo, tanto para o ortodontista quanto para o paciente (Phan \& Ling, 2007).

Em 1997 foi fundada por Zia Chishti e Kelsey Wirth, uma empresa localizada em Santa Clara, California, EUA, chamada Align Technology (Jóias et al, 2011; Neves et al, 2012). Já em 1998 essa empresa conseguiu inovar a ideia de alinhadores ortodônticos removíveis sequenciais que se tinha na época, através da criação do dispositivo Invisalign® que combinava o uso de computadores (sistema CAD-CAM), com técnicas laboratoriais (Phan \& Ling, 2007). Trata-se de uma tecnologia tridimensional computacional que, utiliza modelos estereolitográficos e cálculos algorítmicos, possibilitando movimentar os dentes virtualmente (de $0,15 \mathrm{~mm}$ a $0,25 \mathrm{~mm}$ ) e com isso a criação de uma série de alinhadores sequenciais, que deveriam ser trocados a cada 14 dias partindo apenas de um único molde (Vieira et al, 2013).

A Align Technology obteve resultados considerados satisfatórios para casos com apinhamentos leves e moderados, e desde então, em 1999 o sistema Invisalign ${ }^{\circledR}$ deu origem a novos protocolos clínicos trazendo várias inovações, e depois disso foram surgindo gerações cada vez mais tecnológicas (Neves et al, 2012).

Em 2021 foi lançado o protocolo G8 do Invisalign ${ }^{\circledR}$, no qual se faz o uso do sistema SmartForceAlignActiation que consegue aplicar forças em diferentes áreas dos dentes, aumentando a previsibilidade clínica em movimentos de translação e intrusão, tornando possível a correção de mordidas profundas graves e expansão posterior (Thiensen, 2021). Esse novo sistema apresenta 15 diferentes tipos de attachments para alcançar velocidade máxima de movimento por estagio (Thiensen, 2021).

O foco inicial do Invisalign ${ }^{\circledR}$, era tratar casos mais simples, no entanto, com a evolução dos aparelhos e a possibilidade de se adicionar ferramentas acessórias, como os attachments, tornou-se possível conseguir bons resultados em casos mais complexos, no entanto, essa técnica ainda apresenta algumas dúvidas quanto a sua eficácia (Lonzetti, 2019).

\subsection{Indicações e contraindicações das placas alinhadoras}

Os alinhadores ortodônticos estéticos, são uma excelente opção de tratamento para maloclusões simples em adultos e 
jovens, que são exigentes quanto à estética e não estão dispostos ao tratamento ortodôntico fixo convencional, utilizando-se bráquetes e fios (Vieira et al, 2013).

A rede Invisalign $®$, trata oclusões leves e moderadas, apresentando bons resultados em casos como: apinhamentos leves e moderados (1-5mm); maloclusão dentária leve; problemas de mordida profunda (em especial a má oclusão classe II, divisão 2); diastemas de leves a moderados (1-5mm); recidiva de tratamentos ortodônticos; atresia de arco dental de origem não esquelética (arcos atrésicos que podem ser expandidos sem inclinação dentária excessiva); sobremordida que pode ser reduzida pela intrusão e em casos onde a discrepância do perímetro do arco, associadas a uma discrepância de Bolton, que seriam passíveis de resolução com a exodontia de um incisivo inferior (Joffe, 2003; Neves et al, 2012).

Mesmo que esse sistema possibilite a colocação de attachments, colagem de botões aos dentes e utilização de elásticos intra e intermaxilares, quando o caso exige destes dispositivos e mecânicas mais complexos, não é possível garantir os resultados utilizando tais mecanismos (Boyd, 2005; Joffe, 2003; Neves et al, 2012; Vieira et al, 2013). O sistema Invisaling® algumas vezes consegue tratar alguns casos com dificuldades, causando dúvidas quando a eficácia do tratamento, pode-se citar como exemplos desses casos: apinhamentos e espaçamentos superiores a $5 \mathrm{~mm}$; discrepâncias esqueléticas anteroposteriores superiores a 2mm (medidos pela relação de Classe I); dentes com coroas clínicas curtas; extrusões dentárias; inclinações dentárias superiores a $45^{\circ}$; Dentes com giroversão superior a $20^{\circ}$; mordida aberta anterior e posterior; discrepância entre relação cêntrica e oclusão cêntrica; arcadas com múltiplas perdas dentárias e como contensão no período pós tratamento ortodôntico (Joffe, 2003). No entanto, Phan \& Ling, afirmaram que estes casos podem ser fatores para a não utilização destes aparelhos, devido a sua complexidade e por levarem a um aumento no custo e no tempo de tratamento (Phan et al, 2007).

Vieira et al, afirmaram que tratamento de maloclusões complexas, que necessitem de extrações de pré-molares e incisivos inferiores; distalização de molares; sobremordida acentuada; dentes impactados; pacientes com problemas periodontais e a ausência de conformidade por parte do paciente podem ser exemplos de casos em que a utilização de Invisalign® não é indicada (Vieira et al, 2013).

O uso da técnica dos alinhadores, traz várias vantagens ao paciente. A sua principal vantagem é ser estético, geralmente confeccionado de poliuretano que é um material translucido que se mistura com a cor dos dentes (Jóias et al, 2011). O paciente pode removê-lo para comer, higienizar, ou até mesmo para ir a situações ou eventos especiais (Neves et al, 2012; Jóias et al, 2011; Joffe, 2003). A elaboração dos alinhadores toma uma única impressão no início do tratamento dando comodidade ao paciente (Silva et al, 2017) e a fala geralmente é alterada apenas nas primeiras 24 horas (Joffe, 2003). O paciente depois de comer poderá escovar os dentes normalmente e recolocar os alinhadores, mantendo assim uma boa higienização (Couto \& Abreu, 2020; Lu et al, 2018). A previsibilidade no tratamento, através do qual o paciente consegue ver todo o tratamento antes mesmo de começar (Santos, 2020; Thiesen, 2021). Menos lesões nos tecidos moles, como aftas e mais conforto durante o tratamento as lesões causadas pelos aparelhos (Silva et al, 2017). Atletas que praticam esportes de contato físico, em que uma forte pancada pode causar grandes ferimentos e sangramentos que podem desequilibrar o seu desempenho em uma atividade (Silva et al, 2017). Possibilidade de clareamento dos dentes durante o tratamento (Cordeiro \& Zago, 2019). Conservação da face vestibular dos dentes: por não terem bráquetes colados. Menor risco de perda ou fratura de restaurações ou próteses de porcelana e riscos na estrutura dental: no ato da remoção dos aparelhos fixos (Silva et al, 2017). Consegue beneficiar os pacientes com dores miofasciais e articulações, provocando uma redução destas dores (Neves et al, 2012; Cordeiro \& Zago, 2019). Possibilita a incrementação de ferramentas acessórias para casos mais complexos, como "Attachments", botões e outros; além de ótimo suporte prestado aos ortodontistas (Thiensen, 2021).

Já para o profissional capacitado a utilização da técnica, também apresenta algumas vantagens, como: aumento do público alvo no consultório; os alinhadores estéticos são uma importante ferramenta para pacientes que tem aversão a utilização de aparelhos ortodônticos fixos; possibilidade de melhoria do rendimento e motivação do paciente para uso, gerando o marketing 
positivo (Neves et al, 2012; Silva et al, 2017).

Da mesma forma que temos desvantagens em todas as técnicas ortodônticas e ortopédicas existentes, com os alinhadores não é diferente, existem algumas desvantagens que limitam não só o resultado esperado como também exclui determinados casos ou pacientes do uso da técnica. Tem-se como exemplos desses casos: correção intermaxilar limitada (graves discrepâncias esqueléticas não podem ser tratadas apenas com Invisalign®); não cooperação por parte do paciente, no qual a utilização do aparelho é insuficiente, tornando o tratamento ineficaz; alto custo (hoje o preço é mais elevado em comparação com o tratamento convencional de bráquetes de metal, porém semelhante a um tratamento ortodôntico lingual ou bráquetes auto ligáveis); pode ocorrer ligeira intrusão $(0,25$ a $0,50 \mathrm{~mm})$ de dentes posteriores, no qual será corrigido durante o período de retenção; distúrbios da fala, disfagia, e fluxo salivar aumentado em relação ao uso de aparelhos fixos; e a limitação no controle de movimento radicular e extrusivo além de, pouco controle do fechamento de espaços com adequado paralelismo radicular após extrações (Joffe, 2003).

\subsection{Processo de fabricação dos alinhadores estéticos e seus materiais auxiliares}

O tratamento com o sistema Invisalign®, está voltado apenas para especialista em ortodontia, previamente credenciados ao sistema (Jóias et al, 2011). Como de costume na Ortodontia convencional, também é necessário que o paciente já tenha realizado todo o tratamento restaurador básico, caso seja indicado, além de não apresentar doença periodontal, e a erupção dos segundos molares permanentes deve estar finalizada, antes de iniciar o tratamento com o sistema Invisalign® (Braga, 2015). O processo inicia-se com o ortodontista enviando para a empresa nos EUA o planejamento do caso e os exames digitalizados, através do site Invisalign ${ }^{\circledR}$ para se avaliar a viabilidade do tratamento. Em seguida, dentro de 48 horas, o dentista receberá a resposta por e-mail sobre a viabilidade do caso (Dias, 2018; Braga, 2015).

Caso aceito, as documentações indispensáveis para a continuação do tratamento são: exame radiográfico panorâmico, cefalometria em norma frontal e lateral, moldagem dos arcos com silicone de adição em moldeira plástica fornecida pela empresa, confecção e análise de modelos, registro de mordida em relação centrica em PVC (polivinilsiloxano) e 24 fotos extra e intra bucais, estes arquivos são enviados pelo correio eletrônico para a Align Technology, em Santa Clara, EUA (Dias, 2018; Braga 2015; Neves et al, 2012).

Na empresa, os moldes são digitalizados, para que isso ocorra, os moldes são vazados com gesso e colocados sobre um recipiente e cobertos por epóxi e uretano. Este recipiente é colocado em um scanner destrutivo, onde a lâmina rotativa do scanner faz numerosas passagens sobre os modelos revestidos de epóxi, removendo uma camada fina com cada passagem. Em seguida, um computador ligado ao scanner, monta as informações digitalizadas através das passagens da lâmina para criar um modelo tridimensional das arcadas dentárias e da oclusão do paciente (Lonzetti, 2019; Neves et al, 2012).

Através do CAD (Computer Aided Design), que são os moldes digitalizados e a versão 3D dos arcos e da oclusão do paciente onde permite simular as correções virtualmente, de acordo com o planejamento do ortodontista, em uma fase conhecida como "cuttingprocess", na qual cada dente é separado em unidades geométricas e uma gengiva virtual é colocada ao longo da linha gengival da coroa clínica, para servir como a margem para a fabricação dos alinhadores pelo técnico de ortodontia virtual (VOT), com o Treatsoftware da empresa (Align Technology, Santa Clara, Califórnia) (Lonzetti, 2019; Braga, 2015; Neves et al, 2012).

Os movimentos dos dentes, são encenados de modo que não há interferências oclusais e interproximais, a velocidade dos movimentos está dentro dos parâmetros estabelecidos pela empresa. O número de estágios necessários e consequentemente, o número de alinhadores utilizados, depende da quantidade e da complexidade do movimento (Monguilhott \& Zanardi, 2017). Através das orientações do ortodontista, é realizada uma simulação do final do tratamento "final set-up", a partir desta visualização final do tratamento, é elaborada uma sequência de movimentações para atingir esse objetivo "stagingprocess" 
(Lonzetti, 2019; Neves et al, 2012).

Esse planejamento virtual auxilia o ortodontista a estabelecer os limites dos tratamentos, estudando até dois tipos de tratamentos para um paciente e deste modo, podendo optar através da sua experiência clínica pelo melhor tratamento para cada caso (Kravitz, 2009).

O resultado de todo processo é o arquivo virtual "ClinCheck", o qual será enviado pelo VOT ao ortodontista que irá receber o arquivo em seu computador, sendo assim poderá revisá-lo sugerindo alterações ou mesmo concordando com o protocolo de tratamento proposto para que os alinhadores possam ser fabricados (Dias, 2018; Neves et al, 2012). E poder estudar também o grau de complexidade dos movimentos dentários que irá ditar a quantidade de estágios necessários para resolução do tratamento, assim como o custo desta movimentação dentária de forma pausada, estágio por estágio, ou contínua sequencial, como uma animação do tratamento (Lonzetti, 2019; Neves et al, 2012).

Após a aprovação do "ClinCheck", uma nova etapa do planejamento se inicia, o Computer Aided Manufacturing (CAM), ao materializar as informações virtuais do planejamento, produz por meio de estereolitografia, uma série de modelos de resina referentes a cada etapa do tratamento para realizar a movimentação dentária (Braga, 2015). A partir dos modelos de resina, produz-se uma série de alinhadores estéticos transparentes, à vácuo, em uma máquina de moldagem por pressão Biostar (GreatLakesOrthodontic Products, Tonawanda, NY) (Lonzetti, 2019; Neves et al, 2012). A empresa garante movimentação dental lenta (movimento linear: 0,25 mm/mês e angular: 2\% $/$ ês) e sem interferências oclusais ou interproximais (Braga, 2015). Portanto, a complexidade dos movimentos necessários é que determina o número de alinhadores e o custo para cada caso (Lonzetti, 2019; Neves et al, 2012). Esses alinhadores possuem 0.30"' de espessura e permitem de 0,25 a 0.33mm de movimento dentário a cada troca (Lonzetti, 2019; Vieira et al, 2013; Neves et al, 2012).

Os engenheiros da Align Technology formularam um material próprio para uso nos alinhadores, o poliuretano (Lonzetti, 2019; Neves et al, 2012). Os alinhadores são aparados e gravados a laser com as iniciais do paciente, o número do caso, o número do alinhador e o arco (superior ou inferior) (Lonzetti, 2019; Neves et al, 2012; Vieira et al, 2013). Eles são então desinfectados, embalados e enviados para o consultório odontológico. Após 45 dias da aprovação do "ClinCheck", o ortodontista recebe o kit com os alinhadores (Lonzetti, 2019; Braga, 2015; Vieira et al, 2013; Neves et al, 2012).

Deve-se ressaltar também, o cuidado por parte do ortodontista em manter a mesma disposição dentária do momento da moldagem até a instalação do primeiro alinhador, podendo optar por aparelhos de contenção para se conseguir essa estabilidade, pois, se for alterada tal disposição, consequentemente, o alinhador não irá adaptar-se; resultando no insucesso do tratamento pela falta de continuidade do mesmo (Lonzetti, 2019; Neves et al, 2012; Jóias, 2011). Quanto mais eficiente a reprodução de uma imagem virtual próxima à realidade, mais eficaz será a adaptação e eficiência dos alinhados (Lonzetti, 2019; Vieira et al, 2013; Neves et al, 2012).

Em casos onde extrações dentárias prévias são indicadas, os dentes podem ser extraídos antes da moldagem, mantendo o espaço com a ajuda das contenções ou também extraídos virtualmente (Lonzetti, 2019; Neves et al, 2012; Vieira et al, 2013).

\subsection{Previsibilidade e acompanhamento longitudinal}

Uma das maiores vantagens do tratamento ortodôntico com alinhadores é a possibilidade de escolher como será o movimento de cada dente, no tratamento com Invisalign® isso é feito com software clincheck (Santos, 2020). O sistema Invisalign ${ }^{\circledR}$ apresenta um método de planejamento virtual bastante eficaz, no entanto, nem sempre o planejamento virtual final do sistema, apresentará o mesmo resultado no paciente, pois o sistema apresenta algumas limitações e não realiza por completo alguns movimentos dentários (Kuczynski, 2016).

Através de um estudo com 37 pacientes, foi avaliado a precisão dos movimentos durante o tratamento com Invisalign® e conseguiram afirmar que precisão média para movimentos em todos os dentes foi de $41 \%$. No entanto constataram que existe 
uma enorme variação na precisão com este tratamento, através do qual, conseguiram uma taxa de precisão de 59,3\% na constrição lingual dos caninos inferiores e uma taxa de 18,3\% para extrusão maxilar (Kravitz et al, 2020).

Um estudo com modelos digitais de 14 pacientes, também avaliaram a previsibilidade no tratamento com Invisalign® nos movimentos de rotação, angulação e inclinação e concluíram que no geral este aparelho apresenta uma previsibilidade de moderada a baixa. Ficou comprovado que se tem uma boa previsibilidade para: rotação, inclinação e angulação de incisivos; rotação e angulação de caninos inferiores e inclinação de caninos; rotação de primeiros molares e de segundos molares superiores. No entanto, este aparelho apresenta baixa previsibilidade para angulação de caninos superiores; rotação de pré-molares inferiores; angulação dos pré-molares superiores; inclinação dos molares e angulação dos segundos molares, com isso, cabe ao ortodontista fazer algumas sobrecorreções no planejamento de movimentos com baixa previsibilidade (Santos, 2020).

\section{Discussão}

Apesar de tanto os tratamentos ortodônticos convencionais quanto os realizados utilizando alinhadores invisíveis serem confiáveis e eficientes, foi mostrado pela literatura que cada um possui suas próprias indicações onde se desempenha melhor.

Foi constatado por Couto e Abreu, (2020), que pacientes submetidos a tratamentos com alinhadores invisíveis relatam uma maior ocorrência de dor após decorridos os primeiros 4 dias do tratamento e consequentemente consumiram uma maior quantidade de analgésicos, o que leva ao questionamento se o benefício estético compensa esse aumento de dor e de consumo de medicamentos. Mas não se pode deixar de lado que a dor durante as trocas de alinhadores são menores do que as dores pós manutenção do aparelho convencional.

Ainda nos estudos de Couto e Abreu, (2020), foi observado que os pacientes que preferiram se submeter a tratamentos utilizando-se dos alinhadores invisíveis, demonstraram uma saúde gengival melhor a curto prazo em comparação com os pacientes que optaram pelo tratamento ortodôntico convencional com aparelhos fixos, no entanto quando observado o estado da saúde periodontal a longo prazo, não foi encontrado nenhuma diferença significativa entre os dois tratamentos. Para Couto e Abreu, (2020) e Lonzetti, (2019), este fato deixa claro que ao se deparar com um caso que pode ser tratado tanto com alinhadores invisíveis como por aparelhos fixos convencionais, o quesito saúde periodontal não é o melhor para auxiliar na escolha e mais uma vez os pontos que possuem maior peso no momento da escolha é a estética ainda imbatível dos alinhadores e conforto.

Também é possível observar, ao analisar a literatura disponível, que pacientes que utilizam aparelhos fixos convencionais demonstram menor dificuldade para realizar atividades gerais em comparação aos alinhadores, no entanto no momento de se alimentar os alinhadores se sobrepõem uma vez que estes podem ser removidos durante a alimentação proporcionando um maior conforto aos pacientes, tal fato pode ser observados em estudos como o de Couto e Abreu (2020).

Apesar dos alinhadores invisíveis terem se mostrados eficientes no tratamento de maloclusões simples, foi demonstrado nos trabalhos de Phan e Ling, (2007) e Lonzetti (2019), que estes encontram sérias dificuldades para alcançar resultados semelhantes aos entregues pelos aparelhos convencionais fixos, o que por sua vez acaba gerando maiores gastos nos tratamentos devido ao maior tempo necessário para chegar a resultados semelhantes aos fornecidos pelos aparelhos convencionais e ainda ao final do tratamento o resultado obtido pode não ser exatamente o esperado uma vez que os alinhadores invisíveis não possuem uma previsibilidade tão eficientes, fato que é demonstrado em diversos estudos.

Quando observamos o emprego dos alinhadores dentro de suas indicações, como é demonstrado nos estudos de Jóias et al, (2011) e Lonzetti (2019), notamos que estes podem ser considerado uma excelente escolha proporcionando estética, conforto, facilidade de higienização oral e redução do tempo de trabalho, no entanto é importante voltar ressaltar que este sistema não é indicado para todos os casos, este apresenta seus melhores resultados em casos de maloclusões simples, em caso mais complexos os aparelhos ortodônticos convencionais continuam demonstrando resultados superiores.

Apesar de o tempo preconizado para troca de cada par de alinhadores serem de 15 dias, diversos estudos como o de 
Lonzetti 2019, observaram que este tempo vem se mostrando insuficiente em diversos casos para a realização de movimentos dentários eficientes, tal fato aumenta as chances de ocorrer recidivas, o que acaba fazendo com que os ortodontistas deem uma maior preferência aos tratamentos com aparelhos fixos convencionais.

Vale ressaltar que segundo Lonzetti (2019), a previsibilidade fornecida pelo sistema ClinCheck® dos alinhadores não vem demonstrando $100 \%$ de eficiência, o que acaba aumentando os questionamentos por parte dos ortodontistas, sobre o real custo/benefício destes aparelhos ortodônticos. Ressaltamos ainda que os pacientes que realmente fecham o tratamento para utilização destes dispositivos, são pessoas com poder aquisitivo maior e que apresentam uma exigência estética maior, onde muitos dos questionamentos e procura iniciais pelo tratamento estão voltados para correções de posição e inclinação dentárias mínimas e específicas, o que exige maior refinamento nas técnicas de movimentação ortodôntica.

\section{Conclusão}

Após analisar todo o material utilizado como base bibliográfica, concluiu-se que, os alinhadores invisíveis constituem uma grande evolução para a área da ortodontia, demonstrando estes resultados satisfatórios para os casos que se encaixam dentro de suas indicações, entretanto é recomendado prudência ao ortodontista no momento de realizar sua indicação, uma vez que existe diversos estudos os quais demonstram as dificuldades deste sistema em realizar certos movimentos dentários, o que por sua vez acaba comprometendo sua previsibilidade em alguns casos.

Recomenda-se a realização de novos estudos para investigar a previsibilidade, o que por sua vez incentivará melhorias neste sistema tornando-o ainda mais eficiente e confortável tanto para o profissional quanto para o paciente, além do acompanhamento longitudinal dos pacientes que utilizaram tais alinhadores.

\section{Referências}

Boyd, R. L. Surgical-Orthodontic Treatment of two Skeletal Class III Patients with Invisalign and Fixed Appliances. (2005). J clinorthod. 39(4), 245-257.

Braga, V. C. A. (2015). Sistema Invisalign®: uma alternativa ortodôntica sem bráquetes e fios. Monografia (Pós-Graduação em Ortodontia). Faculdade de Pindamonhangaba, Pindamonhangaba, SP, Brasil.

Cardoso, P. C., Espinosa, D. G., Mecenas, P., Flores-Mir, C., \& Normando, D. (2020). Pain level between clear aligners and fixed appliances: a systematic review. Prog Orthod. 21(1), 1-17.

Chhibber, A., Agarwal, S., Yadav, S., Kuo, C. L., \& Upadhyay, M. (2018). Which orthodontic appliance is best for oral hygiene? A randomized clinical trial. Am J Orthod Dentofacial Orthop. 153(2), 175-183.

Christou, T., Abarca, R., Christou, V., \& Kau, C. H. (2020). Smile outcome comparison of Invisalign and traditional fixed-appliance treatment: a case-control study. Am J OrthodDentofacialOrthop. 157(3), 357-364.

Cordeiro, J. V. C. (2020). Estudo comparativo entre Aparelhos Ortodônticos Fixos e Alinhadores Removíveis: Revisão de Literatura. Trabalho de Conclusão de Curso. Universidade Federal de Santa Catarina, Florianópolis, SC, Brasil.

Couto, B. L. B., \& Abreu, L. G. (2020). Comparação entre alinhadores ortodônticos e aparelhos ortodônticos fixos convencionais: uma revisão sistemática e meta-análise. Arq Odontol. 56(30), 1-35.

Dias, G. (2018). ALINHADORES INVISÍVEIS: algumas opções - Invisalign®, Clear Aligner®e Essix®Clear Aligner. Monografia (Especialização). Faculdade Sete Lagoas, Sete Lagoas, MG, Brasil.

Estrela, C. (2018). Metodologia Científica: Ciência, Ensino, Pesquisa. Editora Artes Médicas.

Joffe, L. (2003). Invisalign: early experiences. J Orthod. 30(4), 348-352.

Jóias, R. P., Sanders, D., Cepera, F., Paranhos, L. R., \& Torres, F. C. (2011). Aparelhos ortodônticos sequenciais removíveis - considerações gerais e apresentação de caso clínico. Rev fac odontol. 16(3), 332-336.

Kesling, H. D. (1945). The philosophy of tooth positioning appliance. Am J Orthod. 31(6), 297-304.

Kravitz, N. D., Kusnoto, B., BeGole, E., Obrez, A., \&Agran, B. (2020). How well does Invisalign work? A prospective clinical study evaluating the efficacy of tooth movement with Invisalign. Am J OrthodDentofacialOrthop. 135(1), 27-35.

Kuczynski, A. (2016). Alinhadores Invisalign-Um Relato De Caso Clínico. Trabalho de Curso. Universidade Federal do Paraná, Curitiba, PR, Brasil. 
Research, Society and Development, v. 10, n. 10, e260101018557, 2021

(CC BY 4.0) | ISSN 2525-3409 | DOI: http://dx.doi.org/10.33448/rsd-v10i10.18557

Lonzetti, M. (2019). Alinhadores do Sistema Invisalign®: fundamentos e aplicação na Ortodontia. Trabalho de Conclusão de Curso. Universidade Federal de Santa Catarina, Florianópolis, SC, Brasil.

Lu, H., Tang, H., Zhou, T., \&Kang, N. (2018). Assessment of the periodontal health status in patients undergoing orthodontic treatment with fixed appliances and Invisalign system: a meta-analysis. Med (Baltimore). 97(13), 1-10.

McNamara, J. A., Kramer, K. L., \&Juenker, J. P. (1985). Invisible Retainers. J clin orthod. 19(8), 570-8.

Monguilhott, L. M.J., Zanardi G. (2017). Tratamento ortodôntico com o sistema Invisalign: a utilização de alta tecnologia na realização de movimentos dentários. Rev Clín Ortod Dental Press. 16(1),56-73.

Neves, C. P. T., Coutinho, I. L., Ferreira, E. A., Coutinho, T. L., \& Miranda, S. C. C. (2012). Sistema Invisalign®: uma alternativa ortodôntica estética. Pós Rev. 6.

Noble, J., Hechter, F. J., Karaiskos, N. E., Lekic, N., \&Wiltshire, W. A. (2009). Future practice plans of orthodontic residents in the United States. Am J OrthodDentofacOrthop. 135(3), 357-360.

Phan, X., \& Ling, P. H. (2007). Clinical Limitations of Invisalign. J Can Dent Assoc. 73(3), 263-266.

Ponitz, R. J. Invisible retainers. (1971). Am J Orthod. 59(3), 266-272.

Santos, R. F. (2020). Previsibilidade da movimentação ortodôntica com alinhadores e metodologia para mensuração do posicionamento dentário em modelos digitais. Tese. Univerisdade de São Paulo, Faculdade de Odontologia, São Paulo, SP, Brasil.

Thiesen, G. (2021). Novo protocolo clinico do Invisalign G8. Aligners Expert.

Vieira, G. M., Franco, E. J., \& Guimarães Junior, C. H. (2013). Alinhadores invisíveis: indicações, limitações biomecânicas e a problemática da mensuração das forças aplicadas. Rev ClínOrtod Dental Press. 12(1), 94-104.

White, D. W., Julien, K. C., Jacob, H., Campbell, P. M., \& Buschang, P. H. (2017). Discomfort associated with Invisalign and traditional brackets: a randomized, prospective trial. AngleOrthod. 87(6), 801-808. 